\title{
Correlational Analysis of Brain Waves According to Sensual Evaluation on Commercial Makgeolli
}

\author{
Sung-Ho Kim ${ }^{1}$, Yong-Suk Kim ${ }^{2}$ and Yong-Sung Kim ${ }^{1 *}$ \\ ${ }^{1 *}$ Next-Generation Converged Information Services Technology Center, \\ Department of Computer Engineering, Chonbuk National University \\ ${ }^{2}$ Department of Food Science and Technology, Chonbuk National University \\ \{marsho21,kimys08,yskim\} @jbnu.ac.kr
}

\begin{abstract}
There are many adjectives to express the taste of Korean Makgeollis. In the paper, firstly those adjectives are used to categorize the Makgeollis based on rough tolerant relation as the same way as document clustering and secondly, Makgeollis which are for domestic marketing were selected and classified according to physicochemical, microbiological analysis and sensual evaluation. We researchers had a lot of difficulties in determining the taste properties of Makgeollis which seem to reflect each regional character. The reason could be that most of Makgeollis are produced through the standardization of taste under a mass production.This paper aims to determine the taste properties of Makgeollis which are currently sold in the domestic market. To achieve this, we researchers attempted the correlational analysis of brain waves according to the sensual evaluation data and electroencephalographic data
\end{abstract}

Keywords: Sensual Evaluation, Taste-adjective, Brain Waves, EEG, Makgeolli

\section{Introduction}

To globalize alcoholic beverages which are commercially available, the standardization and measure of taste must be needed. We have already realized the case that European wines enhance their values and improve their productivity through their taste specialization by the brand.

Makgeolli is one of the most often drunk traditional alcoholic beverages in Korea for thousands years. Makgeolli are prepared with rice, wheat flour, corn, barley, sweet potato, and glucose as major raw materials, and fermented with koji or Nuruk[1].

Especially adjectives for sensory evaluation are well developed in Korean language so that there are so many adjective terms that express the taste of Makgeolli. In the previous pilot research based on the mutual similarities of the pairs of terms, we found that it is hard to construct a complete set with small number of adjectives that can generally be used to express all kinds of Korean Makgeolli.

We researchers selected 4 sorts of the country's most representative Makgeollis currently sold in the domestic market. Then, we found out 55 taste-adjectives by means of factor analysis, clustering and multidimensional analysis in order to attempt the correlational analysis. On the basis of the taste-adjectives we analysed the correlation among the subjects of study, 4 sorts of the country's most preferable Makgeollis, using both sensual evaluation and taste sensor. However, since it is rather different to determine a series of properties which Makgeollis have with them, we tried to use the brain computer interface [6]. 
This paper concentrates on, therefore, the elicitation of regional properties of Makgeollis from the taste correlation between the electroencephalographic data of sensual evaluation panel and the data of sensual evaluation.

\section{Related Works}

\subsection{Taste-Adjectives}

The taste is described as sensory reaction caused by the chemical stimulus. To recognize the taste, materials dissolved in saliva stimulate gustatory cells and this signal is delivered to the cerebrum. Thus, it takes 1 minute to taste. After we eat food, its taste stays 30 seconds.

As it is showed in Figure 1 it is generally utilized to represent seven tastes. However, hot taste is closer to cutaneous sense rather than pure sense of taste
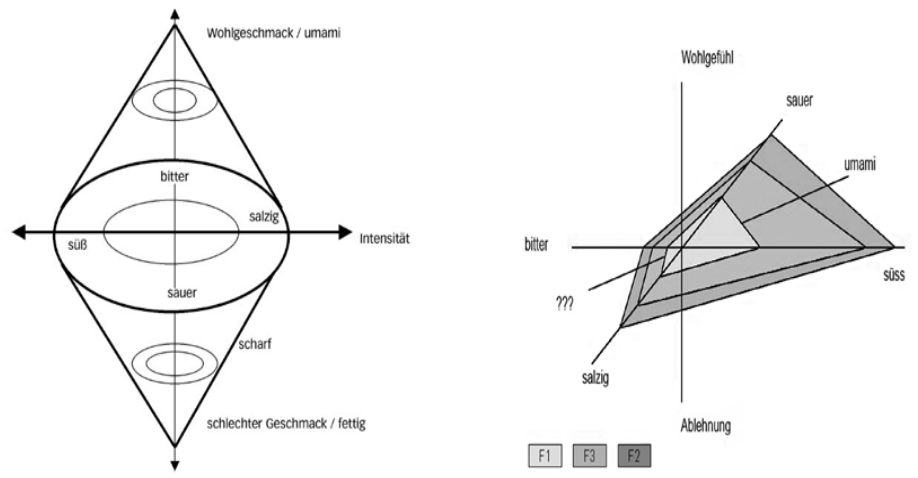

Figure 1. 3-dimensional word field model of taste-adjectives

When the properties of Makgeollis is classified, we have to consider the point: Although a certain taste is generally classified as good, it can be recognized as unpleasant if it shows more than a specific concentration.

\subsection{Taste and Sensual Evaluation}

The sensual evaluation is used to measure the properties of certain products and identify their differences with the help of human sense organs. For example, wines contain hundreds of chemicals, some of which influence the determination of wine taste: sweet taste, astringent taste, sour taste etc. It is, however, far more important to use the actual evaluation of human in determining the taste qualities of wine [5].

\subsection{Brain Waves Interface}

Brain waves are the electric flow which is generated when signals are carried into cerebral nerves in the nervous system [2]. They are also called electroencephalogram (EEG). When we measure brain waves, we can obtain a very complicated type of analogue waveform. We can it the raw date of EEG. We transform the raw data into the digital data [3]. Then, the digital data are again transformed into the power spectrum which is actually utilized to analyse the given data. The power spectrum of brain waves is classified according to frequencies. The amplitude of frequencies is different according to the state of mind and body of sensual evaluation panels [7]. 
The shapes of brain waves appear differently according to brain activity degree. The more actively the brain works, the wider the frequency bandwidth of brain waves becomes, as it is demonstrated in Table 1.

Table 1. Type and feature of Brain waves

\begin{tabular}{|l|l|l|}
\hline Type & Frequency & Nomalty \\
\hline \hline$\delta$ (delta) & $0.5 \sim 4 \mathrm{~Hz}$ & hypnoidal \\
\hline$\theta$ (theta) & $4 \sim 7 \mathrm{~Hz}$ & slow wave sleep \\
\hline$a$ (alpha) & $8 \sim 12 \mathrm{~Hz}$ & stable wave \\
\hline Mid- $\beta$ (mid beta) & $16 \sim 20 \mathrm{~Hz}$ & concentrate stable wave \\
\hline$\beta$ (beta) & $21 \sim 30 \mathrm{~Hz}$ & action stress wave \\
\hline$\gamma$ (gamma) & $30 \sim 50 \mathrm{~Hz}$ & arousal and excitement \\
\hline
\end{tabular}

The shapes of brain waves appear differently according to brain activity degree. The more actively the brain works, the wider the frequency bandwidth of brain waves becomes, as it is demonstrated in Table 1. The power spectrum analysis is the most used method in the analysis of brain waves. For this study the power spectrum analysis is mostly utilized because it is the most adequate method for the time series frequency analysis of the raw data varying according to time.

\section{Correlational Taste-Analysis of Sensual Evaluation and Brain Waves}

\subsection{Collection of Representative Taste-Adjectives}

There are several advance works before the sensual evaluation of 4 sorts of Makgeollis. Firstly, regional Makgeollis are selected. Secondly, 20 students find 87 taste-adjectives using Korean dictionaries and Internet. Thirdly, only 55 of 87 collected taste-adjectives is finally selected through 4 contrast analyses. Fourthly, by means of factor analysis, applied clustering algorithm and multidimensional analysis we make 10 selected taste-adjectives be disposed of by statistical treatment. The result of these procedures is showed in Figure 2.

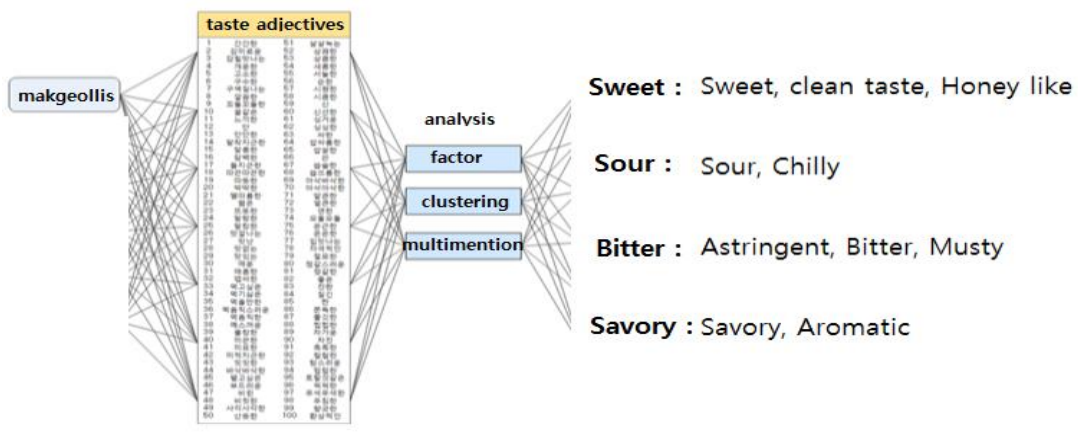

Figure 2. Makgeollis-Adjectives

As the result of the experiment we get four representative Makgeollis-adjectives that describe the taste of Makgeolli: sweet, sour, bitter, savory. 


\subsection{Sensual Evaluation}

We researchers bought 8 Makgeollis which are currently sold in the domestic market. In addition to the sensual evaluation we analyzed the taste using the taste sensor TS-5000Z that was produced in Japan. the taste sensor has a similar structure as the taste-buds in the human tongue. It senses taste elements, transforms the information into electrical signals and transfers the signals into the collected computer. The computer changes the input signals into available data

In this paper the Makgeollis from Chonra-Do were selected as a standard taste reference. On the basis of that other Makgeollis were evaluated. Figure 3. is the analysis material of taste sensor and sensual evaluation

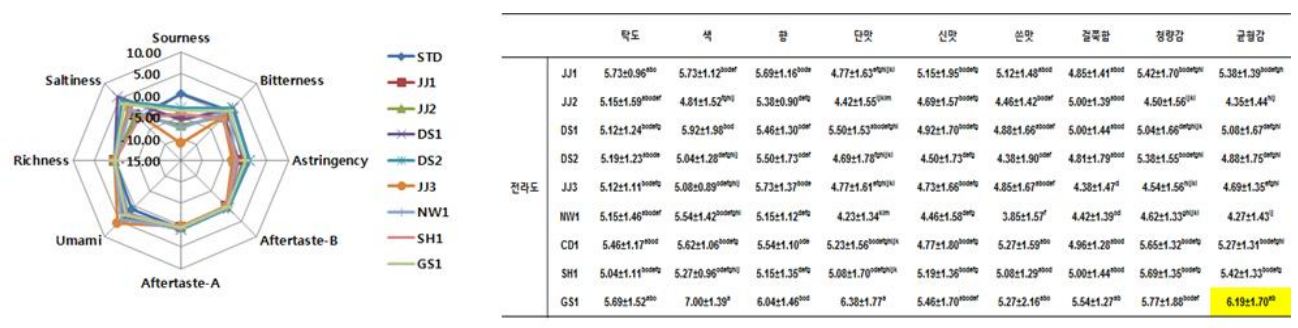

Figiure 3. Analysis of Taste Sensor and Sensual Evaluation of the Makgeollis from ChonraDo

\subsection{Brain Waves Detection}

The electroencephalograph we researchers used was produced by the Neuro in America. The Mindset-Electroencephalograph has a series of advantages: Firstly, it can distinguish between attention and meditation. Secondly, it can analyze brain waves in real time in that it uses the neuro feedback system. Thirdly, it can make the power spectrum of necessary parts.

The analogue data obtained from the raw data of brain waves are transformed into the digital data in the form of the power spectrum through the Fast Fourier Transform(FFT). Then, the digital data is input in Excel and analyzed. The FFT is a kind of algorithm that transforms the frequency field according to time into digital data. The digital process of brain waves is below in Figure 4.

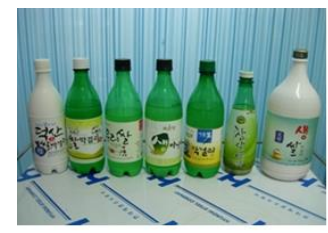

a) makgeolli

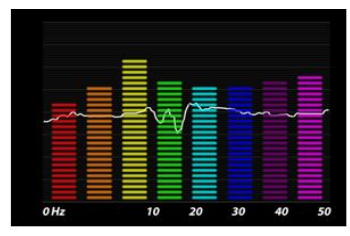

b) raw data

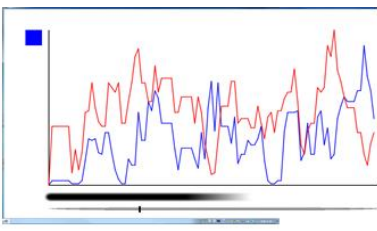

c) power spectrum

Figure 4. Transformation Process

The power spectrum analysis is a kind of analysis technique that is used when the time-series signals changing according to time is transformed into the frequency field and the signal aspect is evaluated by the change degree of the frequency.

As time goes by, the numerical value of the subjects towards the selected Makgeollis can be changeable. To register this sort of change we researchers measured the brain waves of the subjects 10 times per minute after drinking a glass of Makgeolli. At that time, the 5-scaled 
adaptation value was used. Figure 5. shows the analysis results of 4 Makgeollis from ChonraDo.
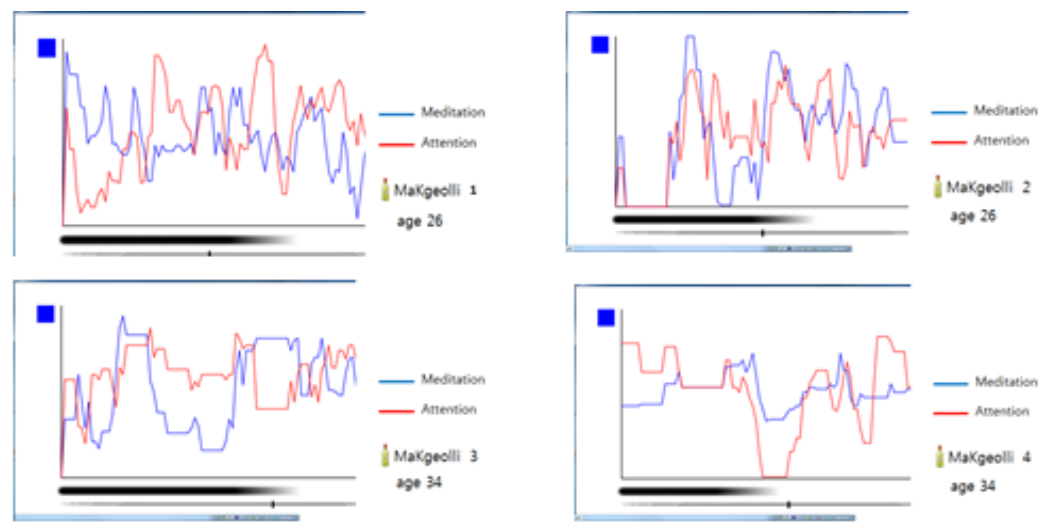

Figure 5. Analysis Results of 4 Makgeollis from Chonra-Do

As it is demonstrated in Figure 5, the higher the numerical values are, the higher the preference of sensual evaluation subjects for the relevant Makgeolli is.

\section{Conclusion}

There are many adjectives to express the taste of Korean Makgeolli. In the paper, those adjectives are used to categorize the Makgeolli based on rough tolerant relation as the same way as document clustering.

To analyze the properties of 4 Makgeollis which are currently sold in the domestic market, we researchers attempted to analyze the raw data of brain waves varying according to drinking time of the sensual evaluation subjects. Through this we researchers find that the $\alpha$-wave form $(8 \sim 12 \mathrm{~Hz})$ indicates the high preference of Makgeolli and the Mid- $\beta$-wave or $\beta$-wave signifies the low preference of Makgeolli, respectively.

For this paper we conducted the experiment using only four subjects between 25 and 35 years of age.

In the future, however, our another experiment is expected to be conducted with much more subjects to have the probability and reliability of statistics.

Above all, we will involve the effect of colour and smell on taste in our research field.

\section{Acknowledgements}

This research was supported by Basic Science Research Program through the National Research Foundation of Korea (NRF) funded by the Ministry of Education, Science and Technology [No. : 2012S1A5B6034452].

\section{References}

[1] H. -E. Kim, "Correlation between Analytical Values of Physicochemical and Sensory Characteristics and Taste Sensing System on Commercial Makgeolli", International Workshops on FRS, (2013) December 11-13; Jeju, Korea.

[2] B. Obermaier and G. Pfurtscheller, "Information translate in a five-classes brain-computer Interface", IEEE Trans., vol. 9, (2001). 
[3] J. -S. Ahn, "Using pattern Analysis of EEG Signals for Implementation of Game Interface", Graduate School of ChungAng University, (2010).

[4] N. C. Lang, "A tolerance rough set approach to clustering web search results", Master thesis, Faculty of Mathematics, Information and Machines, Warsaw University, (2003).

[5] H. -M. Kim, "Comparative Analysis of Multi-Cultural Situations Between Korea and Germany", International Workshops on FRS, (2013) December 11-13; Jeju, Korea.

[6] T. Ebrahimi, J. -M. Vesin and G. Garcia, "IEEE Signal Processing Magazine", (2003) January.

[7] J. -Y. Kim, "EEG Signal feature analysis of Smartphone Game User", International Workshops on FRS, (2013) December 11-13; Jeju, Korea.

\section{Authors}

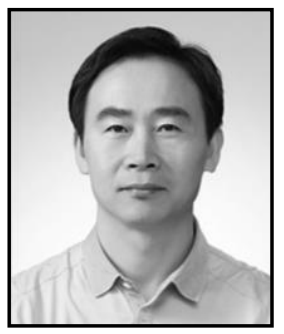

\section{Sung-Ho Kim}

M.D. of Computer Engineering

The doctor's course Computer Engineering

Chonbuk National University

Interest: Computer algorithm, Data mining, Computer game

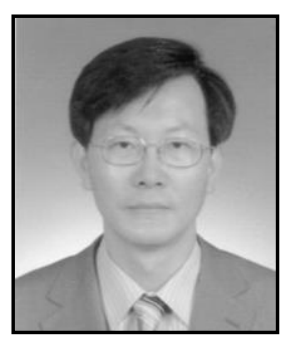

\section{Yong-Suk Kim}

$\mathrm{Ph}$. D. of Food Engineering

Professor, Department of Food Science and Technology,

Chonbuk National University, Republic of Korea

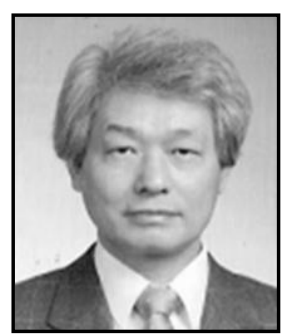

\section{Yong-Sung Kim}

$\mathrm{Ph}$. D. of Computer Engineering

Professor, Department of Computer Engineering,

Chonbuk National University

Chief of Korea convergence society from 2011 to 2013

Interest: Algorithm, Data modeling, Fuzzy system, e-learnning 